\title{
Quantum dash mode-locked lasers for millimeter wave signal generation and transmission
}

\author{
F. van Dijk ${ }^{1}$, B. Charbonnier ${ }^{3}$, S. Constant ${ }^{6}$, A. Enard ${ }^{1}$, \\ S. Fedderwitz ${ }^{2}$, S. Formont ${ }^{6}$, I. F. Lealman ${ }^{5}$, \\ ${ }^{1}$ Alcatel-Thales III-V Lab -Thales III-V Lab, joint lab of \\ 'Bell Labs' and 'Thales Research and Technology', \\ Palaiseau, France \\ frederic.vandijk@3-5lab.fr \\ ${ }^{2}$ Universität Duisburg-Essen, Germany \\ andreas.stoehr@uni-due.de \\ ${ }^{3}$ Orange Labs R\&D, Lannion, France \\ benoit.charbonnier@orange-ftgroup.com
}

\begin{abstract}
In this paper we present the remarkable characteristics of quantum dash mode-locked lasers and how they could be used for low phase noise signal generation, for high data rate wireless transmission and radar in the millimeter wave frequency range.
\end{abstract}

Index Terms: Radio-over-Fiber, Mode-Locked Laser, wireless communications

\section{INTRODUCTION}

Mode-locked laser sources are very attractive solutions for various applications such as, pulse generation, clock extraction from digital data and optical microwave signal generation and processing [1]. It has been observed that devices based on quantum dash (QDs) gain medium emitting in the $1.5 \mu \mathrm{m}$ wavelength range had the property to generate a much narrower beat-note signal linewidth compared with other types of gain media [2]. In this paper we present how this property could be used in different system experiments based on these devices that were performed within the IPHOBAC (Integrated Photonic mm-Wave Functions for Broadband Connectivity) project.

\section{NARROW LINEWIDTH SIGNAL GENERATION WITH QUANTUM DASH MODE-LOCKED LASER}

The different semiconductor laser chips that were used for the system experiments are simple Fabry-Perot cavities with a buried ridge active waveguide. They contain an active layer based on 6 QDs layers grown on InP substrate [2]. Laser cavities of different lengths were cleaved depending on the required mode-locking frequencies: $1715 \mu \mathrm{m}$ for $24.5 \mathrm{GHz}$, $1100 \mu \mathrm{m}$ for $38.5 \mathrm{GHz}, 774 \mu \mathrm{m}$ for $54.8 \mathrm{GHz}$ and $710 \mu \mathrm{m}$ for $58.8 \mathrm{GHz}$. The two remarkable features of these devices is the very wide optical spectrum, with a width exceeding $10 \mathrm{~nm}$ and the very narrow linewidth of the generated beat-note. Figure 1 . presents the beat-note measured on the $54.8 \mathrm{GHz}$ chip with a $3 \mathrm{~dB}$ linewidth of less than $18 \mathrm{kHz}$.

\author{
F. Lecoche ${ }^{3}$, F.Lelarge ${ }^{1}$, D. Moodie ${ }^{5}$, \\ L. Ponnampalam ${ }^{4}$, C. Renaud ${ }^{4}$, M. J. Robertson ${ }^{5}$, \\ A. J. Seeds ${ }^{4}$, A. Stöhr ${ }^{2}$, M. Weiß ${ }^{2}$ \\ ${ }^{4}$ Department of Electronic and Electrical Engineering \\ University College London, London, United Kingdom \\ a.seeds@ee.ucl.ac.uk \\ ${ }^{5}$ CIP Technologies Ltd., Ipswich, United Kingdom \\ dave.moodie@ciphotonics.com \\ ${ }^{6}$ Thales, Elancourt, France \\ stephane.formont@fr.thalesgroup.com
}

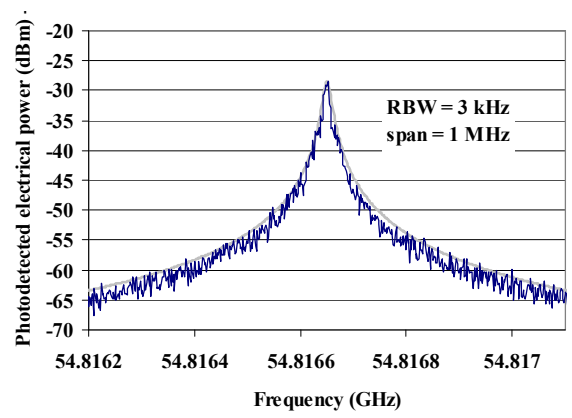

Figure 1. Self pulsation electrical spectrum at $370 \mathrm{~mA}$

III. MILLIMETER WAVE SOURCE INJECTED BY QUANTUM DASH MODE-LOCKED LASER

The $24.5 \mathrm{GHz}$ mode-locked laser was used as an optical frequency comb generator (OFCG) to injection lock a monolithically integrated twin distributed Bragg reflector (DBR) laser [3].

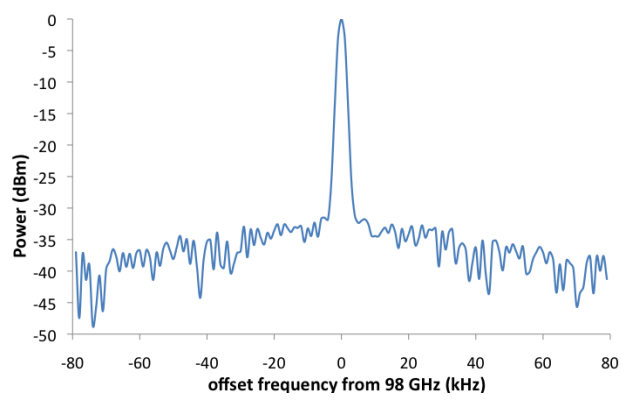

Figure 2. Spectrum of the signal at $98 \mathrm{GHz}$ (Photocurrent : 10mA).

The OFCG has comb lines over a span of $1.6 \mathrm{THz}$, and the twin DBR lasers are designed to give a heterodyne tuning range of $0-1.8 \mathrm{THz}$. Highly coherent millimeter wave ( $\mathrm{mmW})$ signals are generated by locking the twin DBR lasers to 
different comb lines of the OFCG, and then by heterodyning the locked signals in a uni travelling carrier photodiode [4] (Figure 2. The generated signal power at $98 \mathrm{GHz}$ was $0 \mathrm{dBm}$ at a photocurrent of $10 \mathrm{~mA}$ (from $22 \mathrm{~mW}$ optical power), and the $3 \mathrm{~dB}$ linewidth was measured up to $110 \mathrm{GHz}$ and remained $<1$ $\mathrm{kHz}$ over the entire tuning range. The phase noise of the generated signal was $-70 \mathrm{dBc} / \mathrm{Hz}$ at an offset of $10 \mathrm{kHz}$, maintaining the spectral purity of the OFCG. The QDs mode locked laser having comb linewidth of few tens of $\mathrm{kHz}$ have the potential to be integrated with an optical phase lock loop [5] which can be part of a small foot print $\mathrm{mmW}$ source.

\section{HIGH DATA-RATE WIRELESS TRANSMISSION BASED ON A QUANTUM DASH MODE-LOCKED LASER}

\section{A. Indoor Networking}

For high capacity (>1Gbps) radio communications in indoor environments, several standards have been or will be issued to define suitable radio modulations and protocols in the $60 \mathrm{GHz}$ band. In a previous publication [6], we have successfully demonstrated the use of a $55 \mathrm{GHz}$ Quantum Dash ModeLocked Fabry-Perot laser diode for frequency up and down conversion between an intermediate frequency band $(5 \mathrm{GHz})$ and the targeted radio frequency band $(60 \mathrm{GHz})$ for an OFDM type signal carrying $3 \mathrm{Gbps}$ as specified in the IEEE802.15.3c standard. Error Vector Magnitude values of $11 \%$ and $12.8 \%$ where recorded including the distribution of the optical radio signal over $50 \mathrm{~m}$ of Standard Single Mode Fibre.

\section{B. Wireless $H D$}

We also developed a photonic wireless link system based upon the passively mode-locked laser diode for broadband wireless transmission of up to $5 \mathrm{~Gb} / \mathrm{s}$, fulfilling the data rate requirements for uncompressed 1080i and 1080p HDTV as well as for $1 \mathrm{GbE}(1.25 \mathrm{~Gb} / \mathrm{s})$ [7][8].

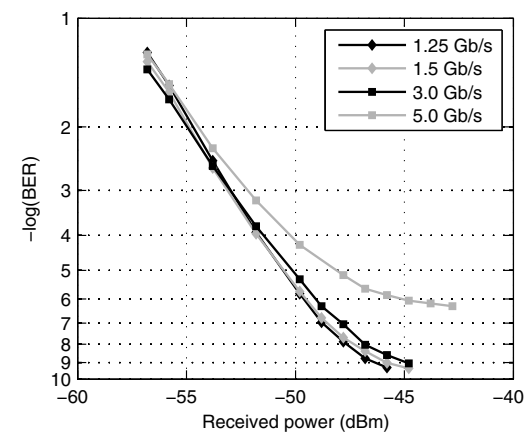

Figure 3. Measured BER as a function of received power after $54 \mathrm{~m}$ fiberoptic and $25 \mathrm{~m}$ wireless transmission for various data rates.

We have performed in- and out-door experiments with a fiber length of $54 \mathrm{~m}$ and wireless path lengths up to $25 \mathrm{~m}$. The receiver sensitivity was experientially determined and is shown in Figure 3. The BER curves for 1.25, 1.5 and $3 \mathrm{~Gb} / \mathrm{s}$ are nearly congruent, exhibiting sensitivities of $-46,-45.5$ and $-45 \mathrm{dBm}$, respectively. The BER curve for $5 \mathrm{~Gb} / \mathrm{s}$-operation shows an error floor at about $10^{-7}$. As can be further seen, error-free transmission of 1.5 and $3 \mathrm{~Gb} / \mathrm{s}$ data signals which fully supports the required data rates for uncompressed 1080i and 1080p HDTV has been achieved. Although applying a modulation scheme with low spectral efficiency, the consumed bandwidth is lower than the allocated unlicensed bandwidth of $57-64 \mathrm{GHz}$, as for the case of the USA thus allowing fully conform operation.

\section{QUANTUM DASH MODE-LOCKED LASERS USED FOR REMOTE SENSING AT $77 \mathrm{GHZ}$}

A $77 \mathrm{GHz}$ optical source has been used in a continuous wave sensor system to detect a moving target. The source was based on a 38.5 GHz mode-locked QDs laser from which the second harmonic was used. The module was integrated in a coupled opto-electronic oscillator configuration similar to previous work [9] in order to reduce the phase noise. The second harmonic was enhanced and the fundamental frequency attenuated thanks to an optimized choice of the fiber spools lengths taking into account dispersion effects in standard single mode fibers. This signal was successfully transmitted over more than $2 \mathrm{~km}$ and used in a mmW radar sensing experiment. Within this frame, successful measurement of the target Doppler has been achieved.

\section{CONCLUSION}

In this paper we have presented how quantum dash modelocked laser could be used in order successfully perform functionalities like: generation of a low phase noise signals at up to $98 \mathrm{GHz}, 60 \mathrm{GHz}$ signal generation, up and down conversion applied to wireless systems, and radar sensing at 77 GHz.

\section{ACKNOWLEDGMENT}

The authors acknowledge the European Commission for its financial support to this work in the frame of the project IST FP6 035317 IPHOBAC (www.ist-iphobac.org).

\section{REFERENCES}

[1] J. Renaudier et al., "Standard-compliant jitter transfer function of alloptical clock recovery at $40 \mathrm{GHz}$ based on a quantum-dot self-pulsating semiconductor laser", IEEE PTL, vol. 18, Iss. 11, 2006 p 1249 - 1251

[2] F. Lelarge et al., "Recent Advances on InAs/InP Quantum Dash Based Semiconductor Lasers and Optical Amplifiers Operating at $1.55 \mu \mathrm{m}$ ", Invited paper, IEEE JSTQE, vol. 13, Iss. 1, 2007 pp. 111 - 124

[3] L. Ponnampalam et al., "Injection-locked integrated twin DBR lasers for mm-wave generation," European Workshop on photonic solutions for wireless, access and in-house networks, Duisburg, Germany, 2009.

[4] E. Rouvalis, C. C. Renaud, D. G. Moodie, M. J. Robertson A. J. Seeds, "Traveling-wave Uni-Traveling Carrier Photodiodes for continuous wave THz generation," Optics Express, Vol. 18, pp. 11105-11110, 2010.

[5] R. J. Steed et al.,"Hybrid integrated optical phase-lock loops for photonic terahertz sources", IEEE J. Select. Topics in QE, in press.

[6] F. Lecoche et al, "60 GHz bidirectional optical signal distribution system at 3 Gbps for wireless home network",. IEEE MWP '09.

[7] A. Stöhr et al, " Millimeter-Wave Photonic Devices for Broadband Wireless Systems", IEEE JLT 2010 (invited paper, to be published)

[8] A. Stöhr et al, "60 GHz Radio-over-Fiber Technologies for Broadband Wireless Services”, Journal of Optical Networking, vol. 8, no. 5, pp. 471-487, 2009, (invited))

[9] F. van Dijk et al "Phase Noise Reduction of a Quantum Dash ModeLocked Laser in a Millimeter-Wave Coupled Opto-Electronic Oscillator", IEEE JLT, Vol. 26, Iss. 15, Aug.1, 2008 pp. 2789 - 2794 